\title{
Optimal liver stiffness measurement values for the diagnosis of significant fibrosis and cirrhosis in chronic liver disease in Singapore
}

Pik Eu Chang ${ }^{1,2}$, MBBS, MRCP, Juanda Leo $\underline{\text { Hartono }}^{3}$, MMed, MRCP, Yee Lin Ngai ${ }^{1}$, BSN, ADM(Nurs), Yock Young $\underline{D a n}^{3}$, MBBS, MRCP, Kieron BL $\underline{\operatorname{Lim}}^{3}$, MBBS, FRCP, Wan Cheng $\underline{\text { Chow }^{1,2}}$, MBBS, MRCP

INTRODUCTION Despite the widespread use of transient elastography for non-invasive assessment of liver fibrosis, the optimal cut-off liver stiffness measurement (LSM) values remain unclear. This study aimed to validate the optimal cut-off LSM values for significant fibrosis and cirrhosis in patients with chronic liver disease (CLD).

METHODS Prospective multicentre data of CLD patients who underwent paired liver biopsy and LSM was analysed to determine the optimal cut-off LSM values for predicting significant fibrosis (METAVIR F $\geq 2$ ) and cirrhosis (METAVIR F4). A high-quality cohort was selected by excluding those with failed LSM and invalid LSM readings.

RESULTS Of the 481 patients recruited, 322 fulfilled the pre-defined quality criteria. CLD aetiology was chronic hepatitis B (CHB) in 49\%, non-alcoholic steatohepatitis (NASH) in $16 \%$ and chronic hepatitis C (CHC) in $12 \%$. Area under the receiver operating characteristic curve for LSM was 0.775 (95\% confidence interval [CI] 0.724-0.826) for significant fibrosis and $0.810(95 \% \mathrm{Cl} 0.738-0.882)$ for cirrhosis. Optimal cut-off LSM values were $9 \mathrm{kPa}$ for significant fibrosis and $13 \mathrm{kPa}$ for cirrhosis in the general cohort. Optimal cut-off LSM values were $9 \mathrm{kPa}$ for significant fibrosis and $12 \mathrm{kPa}$ for cirrhosis for both $\mathrm{CHB}$ and $\mathrm{CHC}$, while the corresponding values for NASH were $11 \mathrm{kPa}$ and $15 \mathrm{kPa}$.

CONCLUSION Optimal cut-off LSM values should be selected based on disease aetiology. In Singapore, the optimal cut-off LSM values for CHB and CHC are $9 \mathrm{kPa}$ for significant fibrosis and $12 \mathrm{kPa}$ for cirrhosis. Optimal cut-off values for $\mathrm{NASH}$ require further validation.

Keywords: cirrhosis, cut-off, fibrosis, liver stiffness, transient elastography

\section{INTRODUCTION}

The assessment of liver fibrosis is integral to the management of chronic liver disease (CLD), as it provides prognostic information for therapeutic intervention to prevent the development of liver-related endpoints, such as cirrhosis, liver failure, liver cancer and death. ${ }^{(1)}$ Although liver biopsy is regarded as the gold standard for fibrosis assessment, it is an invasive procedure that is associated with bleeding risk and is poorly accepted by patients. It is also limited by sampling error and inter-observer variability. ${ }^{(2,3)}$ These factors have led to the development of non-invasive methods for assessment of liver fibrosis and cirrhosis. Of these, liver stiffness measurement (LSM) using transient elastography (TE) has been widely validated as a reliable predictor of fibrosis and cirrhosis in CLD. ${ }^{(4-14)}$

As a testament to the increasing acceptance of TE worldwide, clinical practice guidelines from various international liver associations have endorsed TE as an alternative to liver biopsy for liver fibrosis assessment to aid clinical decision-making in the management of chronic viral hepatitis. ${ }^{(15-17)}$ However, the optimal cut-off LSM values reported in the literature are highly variable, ranging from $5.2 \mathrm{kPa}$ to $9.7 \mathrm{kPa}$ for significant fibrosis and $9.7 \mathrm{kPa}$ to $22.7 \mathrm{kPa}$ for cirrhosis. ${ }^{(17)}$ This wide variability makes it difficult for the practising physician to interpret LSM results for clinical decision-making in the real-world setting.
There are several reasons for the wide variability in cut-off LSM values reported in the literature. Firstly, most validation studies were performed in distinct homogeneous disease populations, resulting in different cut-off LSM values for different diseases. Meta-analysis of TE studies demonstrates that the accuracy of LSM is dependent on the prevalence of the condition in the specific population studied. ${ }^{(5)}$ Secondly, many early studies of LSM were confounded by poor-quality TE readings, inadequate operator experience, obesity, elevated transaminases and suboptimal liver biopsy samples, ${ }^{(18-23)}$ including the first validation study of TE in Singapore. ${ }^{(12)}$ Recent clinical practice guidelines have emphasised that the correct interpretation of LSM in clinical practice must consider the quality of the LSM. ${ }^{(15-17)}$ Attention must also be given to the following: interquartile range/median value (IQR/M); serum transaminase levels; use of the appropriate probe (extra-large [XL] probe if skin-to-capsule distance $>25 \mathrm{~mm}$ ); and absence of clinical conditions known to affect liver stiffness when interpreting LSM. ${ }^{(17)}$

In most hospitals in Singapore, TE is now routinely used to aid physicians in the management of CLD. There is, therefore, an important need to define the appropriate cut-off LSM values that are validated for our local population, based on the prevalence and spectrum of liver diseases that are unique to the Singapore population. Furthermore, these cut-off values must be validated

${ }^{1}$ Department of Gastroenterology and Hepatology, Singapore General Hospital, ${ }^{2}$ Duke-NUS Medical School, ${ }^{3}$ Division of Gastroenterology and Hepatology, National University Hospital, Singapore

Correspondence: A/Prof Chang Pik Eu Jason, Senior Consultant, Department of Gastroenterology and Hepatology, Singapore General Hospital, 20 College Road, Academia, Singapore 169856. jason.chang@singhealth.com.sg 
using reliable, high-quality LSM criteria. To this end, we designed a prospective, multicentre study of high-quality, biopsy-paired TE measurements with the primary aim of defining the optimal cut-off LSM values that are validated in our specific patient population in Singapore for the diagnosis of liver fibrosis and cirrhosis. The secondary aim was to define the optimal cut-off LSM values for specific aetiologies of liver disease.

\section{METHODS}

We reviewed prospectively collected data of biopsy-paired TE from two tertiary hepatology centres in Singapore. This data was collected from the time TE was available in the individual centres. Consecutive patients who underwent liver biopsy and LSM were recruited for the study. Liver biopsies were performed for clinical indications, including staging of fibrosis for therapeutic decisions in patients with chronic viral hepatitis, prognostication in patients with non-alcoholic steatohepatitis (NASH), and diagnostic confirmation of patients with suspected autoimmune hepatitis, primary biliary cirrhosis or unexplained sustained abnormalities in liver function. Patients with decompensated liver cirrhosis and those with hepatocellular carcinoma were excluded from the study. The institutional review boards of the respective institutions approved the study.

Demographic, biochemical, anthropometric and clinical data of each patient was prospectively collected prior to the liver biopsy. Variables recorded included age, gender, race, aetiology of liver disease, body mass index (BMI), baseline liver function tests, serum creatinine and haematological indices. Liver biopsies were performed via the percutaneous, transjugular or surgical routes based on clinical indication and standard clinical practice. Liver specimens were stained according to standard histopathology practice for the assessment of liver fibrosis. Severity of fibrosis was staged by an experienced histopathologist from each centre and standardised across the two centres using the METAVIR scoring system. ${ }^{(24)}$

LSM was performed by experienced operators who had each completed more than 100 examinations. Each measurement was performed after a three-hour fast over the right lobe of the liver, with the patient lying in the supine position with the right arm abducted. At least ten valid LSM readings were obtained and the median LSM reading was recorded. Inability to obtain any valid readings was considered an LSM failure. Measurements with $<10$ readings, $<60 \%$ success rate and IQR $>30 \%$ of the median LSM (i.e. IQR/M>0.3) were deemed to be invalid. Where the $\mathrm{XL}$ probe was available, it was used for LSM in patients with skin-to-liver capsule distance $\geq 25 \mathrm{~mm}$ or BMI $>28 \mathrm{~kg} / \mathrm{m}^{2}$.

We adhered to the recommendations of recently published clinical guidelines, ${ }^{(17)}$ restricting the study population to a high-quality cohort by excluding known confounders. All LSM failures, invalid LSM readings, patients with BMI $>30 \mathrm{~kg} / \mathrm{m}^{2}$, liver biopsy specimens $<15 \mathrm{~mm}$ in length and patients with significant transaminitis (alanine aminotransferase $[\mathrm{ALT}]>5 \times$ the upper limit of normal [ULN]) were excluded. This limited the number of falsely elevated LSM values and provided the most reliable cut-off LSM values for clinical use.
The primary outcome of the study was to define the optimal LSM values for the diagnosis of significant liver fibrosis (METAVIR $\geq$ F2) and cirrhosis (METAVIR F4) in a selected high-quality cohort. The diagnostic performance of TE was assessed by the C-statistic (with 95\% confidence interval $[\mathrm{CI}])$, which was estimated from the area under the receiver operating characteristic curve (AUROC), and the result was compared against the aspartate aminotransferase-to-platelet ratio index (APRI), a well-validated serum marker of fibrosis. ${ }^{(25)}$ We reported optimal LSM values for the diagnosis of significant fibrosis and cirrhosis based on maximal diagnostic accuracy (maximum sum of sensitivity and specificity), maximal sensitivity (sensitivity $>90 \%$ ) and maximal specificity (specificity $>90 \%$ ). We then examined the optimal cut-off LSM values for individual aetiologies of liver disease based on a similar methodology. Qualitative and quantitative differences among groups were compared using chi-square test (or Fisher's exact test where applicable) and Student's $t$-test (including one-way analysis of variance where applicable), respectively. A two-sided p-value $<0.05$ was deemed to be statistically significant. Data in the text and tables was represented as mean \pm standard deviation or number (percentage), unless otherwise specified.

\section{RESULTS}

A total of 481 patients were recruited from two tertiary hospitals in Singapore - National University Hospital and Singapore General Hospital. All subjects underwent liver biopsy, which was paired with TE performed within three months of the biopsy. From the overall cohort, we excluded those with LSM failures $(n=24)$, invalid LSM readings $(n=22)$, invalid biopsy specimens $(n=75)$, $\mathrm{BMI}>30 \mathrm{~kg} / \mathrm{m}^{2}(\mathrm{n}=22)$ and ALT $>5 \times \mathrm{ULN}(\mathrm{n}=16)$, resulting in a final cohort of 322 patients who fulfilled the criteria for highquality TE measurements. The baseline clinical characteristics of the study cohort are summarised in Table I.

Liver biopsy was performed via the percutaneous route in $88.8 \%$ of patients, transjugular route in $5.2 \%$ and surgical route in $5.8 \%$. Mean specimen length was $24.1 \pm 6.9 \mathrm{~mm}$. METAVIR data was not reported in ten patients, and was thus only available in 312 patients. METAVIR fibrosis grades were evenly distributed among the various stages (Table II). Almost $45 \%$ of patients had no or mild fibrosis (F0 and F1), while a similar proportion (41\%) had moderate fibrosis (F2 and F3) and 14\% had cirrhosis (F4). The balanced distribution of fibrosis in our cohort thus allowed for reliable assessment of TE performance across the spectrum of fibrosis stages.

Results of TE were available for all 322 patients. Median LSM value was $9.7 \mathrm{kPa}$ (range 3.4-75.0 kPa), while mean IQR was $2.1 \pm 2.3 \mathrm{kPa}$ and median IQR/M was $0.15 \pm 0.07 \mathrm{kPa}$. The distribution of LSM according to METAVIR fibrosis groups is shown in Fig. 1. Median LSM value was found to correlate well with METAVIR fibrosis grade (Spearman's rank correlation coefficient $0.52 ; p<0.001)$. AUROC for TE diagnosis of significant fibrosis (METAVIR $\geq$ F2) was 0.775 (95\% Cl 0.724-0.826, p < 0.001; Fig. 2a). Optimal cut-off LSM values to predict significant fibrosis and cirrhosis are shown in Table III. An optimal cut-off LSM value 
of $9 \mathrm{kPa}$ provided the maximal diagnostic accuracy for diagnosis of significant fibrosis (sensitivity $76.7 \%$, specificity $68.4 \%$; positive predictive value [PPV] $74.9 \%$, negative predictive value [NPV] 69.9\%; and overall correct classification 72.7\%). An optimal cut-off LSM value of $6 \mathrm{kPa}$ provided $>90 \%$ sensitivity but poor specificity at $33.6 \%$ for the prediction of significant fibrosis. Conversely, a cut-off LSM of $13 \mathrm{kPa}$ provided $>90 \%$ specificity with a corresponding sensitivity of $43.6 \%$ for the prediction of significant fibrosis.

AUROC for TE diagnosis of cirrhosis (METAVIR F4) was 0.810 (95\% Cl 0.738-0.882, p < 0.001; Fig. 2b). An optimal cutoff LSM value of $13 \mathrm{kPa}$ provided maximal accuracy for diagnosis of cirrhosis (sensitivity 70.5\%, specificity 78.7\%; PPV 35.3\%, NPV $94.2 \%$ and overall correct classification $77.6 \%$ ). Optimal cut-off LSM values that provided $>90 \%$ sensitivity and $>90 \%$ specificity for diagnosis of cirrhosis were $7.5 \mathrm{kPa}$ and $21 \mathrm{kPa}$, respectively.

Table I. Baseline characteristics of the study cohort $(n=322)$.

\begin{tabular}{|c|c|}
\hline Characteristic & No. (\%) \\
\hline Age $^{*}(y r)$ & $49.4 \pm 12.3$ \\
\hline \multicolumn{2}{|l|}{ Gender } \\
\hline Male & $179(55.6)$ \\
\hline Female & $143(44.4)$ \\
\hline \multicolumn{2}{|l|}{ Aetiology } \\
\hline Chronic hepatitis B & $159(49.4)$ \\
\hline Non-alcoholic steatohepatitis & $51(15.8)$ \\
\hline Chronic hepatitis $C$ & $37(11.5)$ \\
\hline Autoimmune hepatitis & $13(4.0)$ \\
\hline Primary biliary cirrhosis & $10(3.1)$ \\
\hline Cryptogenic cirrhosis & $3(0.9)$ \\
\hline Alcoholic cirrhosis & $1(0.3)$ \\
\hline Others & $48(14.9)$ \\
\hline \multicolumn{2}{|l|}{ Laboratory $^{\dagger}$} \\
\hline Albumin (G/L) & $40.1 \pm 16.6(14.0-49.0)$ \\
\hline Bilirubin $(\mu \mathrm{mol} / \mathrm{L})$ & $19.9 \pm 23.1(4.0-96.0)$ \\
\hline Alanine transaminase (UL) & $67.3 \pm 35.1(14.0-175.0)$ \\
\hline Aspartate transaminase (U/L) & $48.2 \pm 29.3(16.0-187.0)$ \\
\hline $\begin{array}{l}\text { Gamma glutamyl } \\
\text { transaminase (U/L) }\end{array}$ & $107.1 \pm 175.6(11.0-1,139.0)$ \\
\hline Platelets $\left(\times 10^{9}\right)$ & $205.0 \pm 74.0(19.0-434.0)$ \\
\hline Prothrombin time (s) & $11.5 \pm 1.7(9.1-19.8)$ \\
\hline Body mass index ${ }^{\dagger}\left(\mathrm{m} / \mathbf{k g}^{2}\right)$ & $23.9 \pm 2.8(15.0-29.6)$ \\
\hline
\end{tabular}

* Data presented as mean \pm standard deviation. †Data presented as mean \pm standard deviation (range).
For the prediction of significant fibrosis and cirrhosis, TE was found to perform better than APRI. For the prediction of significant fibrosis, AUROC of TE was 0.775 (95\% Cl 0.724-0.826) vs. $0.584(95 \% \mathrm{Cl} 0.521-0.648)$ for APRI. For the prediction of cirrhosis, AUROC of TE was $0.810(95 \% \mathrm{Cl}$ 0.738-0.882) compared to $0.662(0.578-0.746)$ for APRI.

Next, we evaluated the AUROC and optimal LSM values for assessment of significant fibrosis and cirrhosis in individual aetiologies of liver disease (Table IV). Baseline clinical, laboratory and histological characteristics were not significantly different among the different aetiologies, except for a higher ALT in patients with NASH.

Patients with chronic hepatitis $\mathrm{B}(\mathrm{CHB})$ made up a majority ( $n=159$ ) of the cohort. Among CHB patients, $59.7 \%$ had significant fibrosis and $12.6 \%$ had cirrhosis. Optimal cut-off LSM values of $9 \mathrm{kPa}$ for significant fibrosis and $12 \mathrm{kPa}$ for cirrhosis allowed for correct classification in $70.5 \%$ and $77.6 \%$ of $\mathrm{CHB}$ patients, respectively. The optimal cut-off LSM values for chronic hepatitis $\mathrm{C}(\mathrm{CHC})$ were similar at $9 \mathrm{kPa}$ for significant fibrosis and $12 \mathrm{kPa}$ for cirrhosis, which correctly classified $81.1 \%$ and $70.3 \%$ of patients, respectively.

In the NASH cohort, the performance of TE for the prediction of significant fibrosis and cirrhosis fared better than that for the viral hepatitis cohort; the optimal cut-off LSM values were higher. An LSM value of $11 \mathrm{kPa}$ was the optimal cut-off for the prediction of significant fibrosis with a high AUROC of 0.907 , sensitivity of $94.4 \%$, specificity of $76.9 \%$ and a correct classification rate

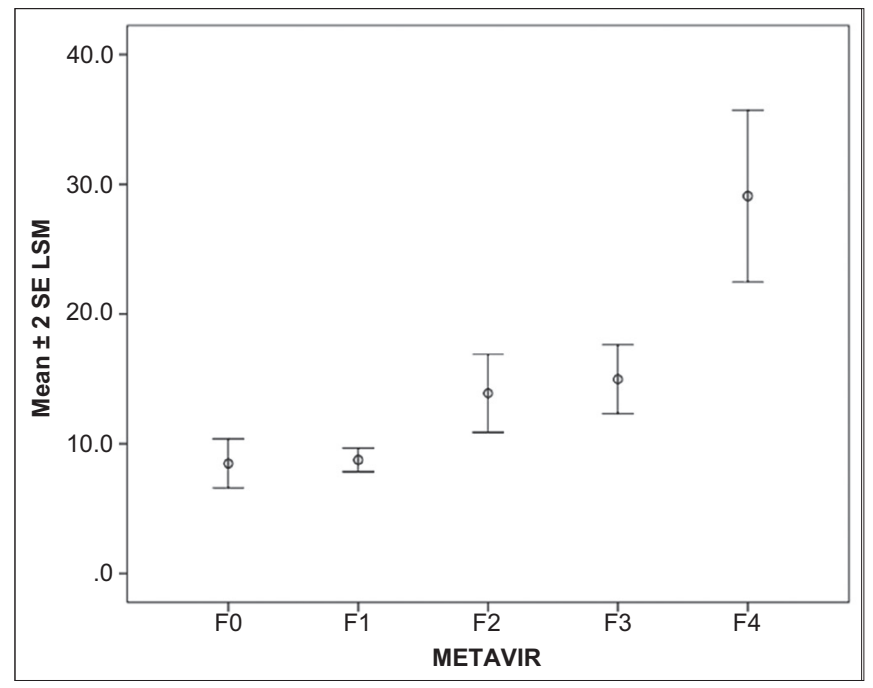

Fig. 1 Graph shows the range of liver stiffness measurement (LSM) values according to the METAVIR fibrosis groups. SE: standard error

Table II. LSM values according to METAVIR fibrosis groups $(n=312)$.

\begin{tabular}{|c|c|c|c|c|c|}
\hline \multirow[t]{2}{*}{ Parameter } & \multicolumn{5}{|c|}{ METAVIR } \\
\hline & Fo & $\mathbf{F 1}$ & $\mathbf{F 2}$ & F3 & F4 \\
\hline No. (\%) of patients & $52(16.7)$ & $88(28.2)$ & $60(19.2)$ & $68(21.8)$ & $44(14.1)$ \\
\hline \multicolumn{6}{|l|}{ LSM (kPa) } \\
\hline Mean \pm SEM & $8.5 \pm 0.9$ & $8.8 \pm 0.5$ & $13.9 \pm 1.5$ & $15.0 \pm 1.3$ & $29.1 \pm 3.3$ \\
\hline Median & 7.1 & 7.8 & 10.3 & 11.9 & 20.9 \\
\hline 25th, 75th quartiles & $5.3,8.9$ & $5.7,10.5$ & $7.7,16.2$ & $9.4,16.8$ & $12.0,40.2$ \\
\hline
\end{tabular}

LSM: liver stiffness measurement; SEM: standard error of mean 

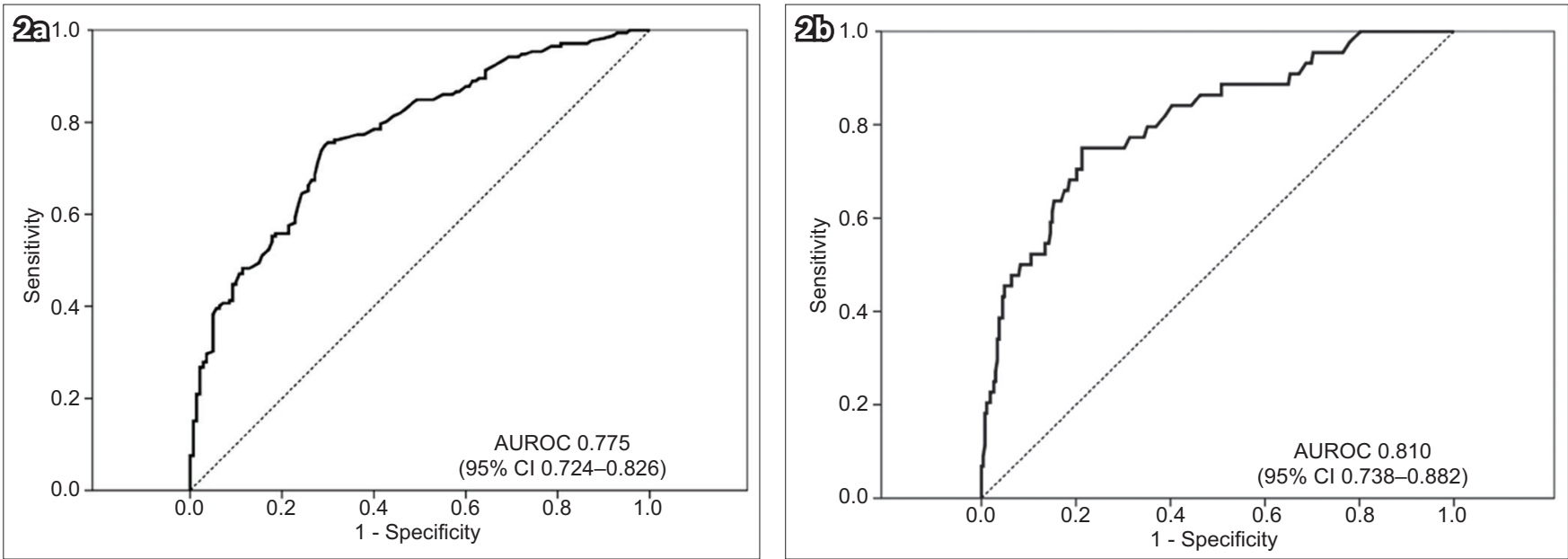

Fig. 2 Graphs show the area under the receiver operating characteristic curve (AUROC) of liver stiffness measurement for the prediction of (a) significant fibrosis (METAVIR $\geq F 2$ ) and (b) cirrhosis (METAVIR F4). Cl: confidence interval

Table III. Optimal liver stiffness measurement values for the prediction of significant fibrosis and cirrhosis.

\begin{tabular}{|lllll|}
\hline Parameter & AUROC & $>\mathbf{9 0 \%}$ sensitivity & Maximal specificity + sensitivity & $>\mathbf{9 0 \%}$ specificity \\
\hline Significant fibrosis (METAVIR $\geq$ F2) & 0.775 & $6.0 \mathrm{kPa}$ & $9.0 \mathrm{kPa}$ & $13.0 \mathrm{kPa}$ \\
\hline Cirrhosis (METAVIR F4) & 0.810 & $7.5 \mathrm{kPa}$ & $13.0 \mathrm{kPa}$ & $21.0 \mathrm{kPa}$ \\
\hline
\end{tabular}

AUROC: area under the receiver operating characteristic curve

Table IV. Optimal cut-off LSM values for the diagnosis of significant fibrosis (METAVIR $\geq$ F2) and cirrhosis (METAVIR F4) in individual aetiologies of chronic liver disease.

\begin{tabular}{|c|c|c|c|c|c|c|}
\hline Aetiology & $\%$ of patients & Cut-off LSM (kPa) & AUROC & Sensitivity (\%) & Specificity (\%) & CC (\%) \\
\hline \multicolumn{7}{|c|}{ CHB $(n=159)$} \\
\hline$\geq \mathrm{F} 2$ & 59.7 & 9 & 0.765 & 67.4 & 75.4 & 70.5 \\
\hline F4 & 12.6 & 12 & 0.759 & 65.0 & 79.4 & 77.6 \\
\hline \multicolumn{7}{|c|}{ CHC $(n=37)$} \\
\hline$\geq F 2$ & 64.9 & 9 & 0.838 & 83.3 & 76.9 & 81.1 \\
\hline $\mathrm{F} 4$ & 16.2 & 12 & 0.621 & 50.0 & 74.2 & 70.3 \\
\hline \multicolumn{7}{|c|}{ NASH $(n=51)$} \\
\hline$\geq F 2$ & 35.3 & 11 & 0.907 & 94.4 & 76.7 & 83.3 \\
\hline $\mathrm{F} 4$ & 11.8 & 15 & 0.950 & 100.0 & 81.0 & 83.3 \\
\hline \multicolumn{7}{|c|}{ PBC $(n=10)$} \\
\hline$\geq F 2$ & 60.0 & 13 & 0.958 & 83.3 & 100.0 & 90.0 \\
\hline $\mathrm{F} 4$ & 40.0 & 24 & 1.000 & 100.0 & 100.0 & 100.0 \\
\hline \multicolumn{7}{|c|}{ AIH $(n=13)$} \\
\hline$\geq F 2$ & 61.5 & 11 & 0.750 & 50.0 & 100.0 & 67.0 \\
\hline F4 & 15.4 & 34 & 0.650 & 50.0 & 90.0 & 83.3 \\
\hline
\end{tabular}

AIH: autoimmune hepatitis; AUROC: area under the receiver operating characteristic curve; CC: correct classification; $\mathrm{CHB}$ : chronic hepatitis B; $\mathrm{CHC}$ : chronic hepatitis C; LSM: liver stiffness measurement; NASH: non-alcoholic steatohepatitis; PBC: primary biliary cirrhosis

of $83.3 \%$. The optimal cut-off LSM value for the prediction of cirrhosis was $15 \mathrm{kPa}$ with an AUROC of 0.950, sensitivity of $100.0 \%$, specificity of $81.0 \%$, and a correct classification rate of $83.3 \%$.

Assessments of the performance of TE in autoimmune hepatitis $(\mathrm{n}=13)$ and primary biliary cirrhosis $(\mathrm{n}=10)$ were limited by the small numbers. For both of these aetiologies, although the optimal cut-off LSM values for prediction of significant fibrosis and cirrhosis were notably higher compared to that for viral hepatitis, the small sample size precluded any meaningful analysis.

\section{DISCUSSION}

This is the first multicentre, quality-controlled, biopsy-paired study of TE performed in Singapore. Our study demonstrated an optimal LSM of $13 \mathrm{kPa}$ for the diagnosis of liver cirrhosis in the overall study cohort with mixed aetiologies. This locally validated cut-off level for non-invasive diagnosis of liver cirrhosis is consistent with those reported in previous publications, which ranged from $10.0 \mathrm{kPa}$ to $14.8 \mathrm{kPa}$. ${ }^{(17)}$ A large meta-analysis assessing the performance of TE reported an optimal cut-off LSM of $13 \mathrm{kPa}$ for the diagnosis of cirrhosis, independent of aetiology of liver 
disease; ${ }^{(5)}$ this result is congruent with the optimal cut-off LSM in our study, and thus validates the excellent performance of TE for non-invasive diagnosis of cirrhosis.

The clinical importance of non-invasive diagnosis of cirrhosis is that it enables reliable detection of early liver cirrhosis in patients with chronic liver disease. Early diagnosis of cirrhosis heralds the need for gastroscopy for varices screening, regular surveillance for hepatocellular carcinoma (HCC), and active treatment of the underlying liver disease to prevent progression to decompensation. ${ }^{(26)}$ Conversely, reliable exclusion of cirrhosis reduces unnecessary invasive endoscopy, cuts down the frequency of HCC surveillance, and may even delay the need for expensive long-term antiviral treatment for $\mathrm{CHB}$. These factors impact patient care by lowering healthcare costs and the utilisation of healthcare resources. Therefore, TE plays a useful clinical role for reliable exclusion of liver cirrhosis, as it performs particularly well to rule out cirrhosis with its high NPV of $94 \%$ irrespective of disease aetiology. TE is now accepted as the most accurate non-invasive method for diagnosing liver cirrhosis. ${ }^{(27)}$ Although ultrasonography (US) is widely used to diagnose liver cirrhosis, there are concerns regarding the overestimation of cirrhosis based on the US finding of coarse echogenicity alone, leading to a false positive diagnosis of liver cirrhosis. ${ }^{(28)}$ In patients with a low pre-test probability of cirrhosis (normal liver function, platelet counts and spleen size) who are diagnosed with early cirrhosis on US, we recommend seeking confirmation with TE. An LSM value $<13 \mathrm{kPa}$ should caution the physician against labelling such patients as cirrhotic. These patients may benefit from liver biopsy to confirm the diagnosis of cirrhosis.

In contrast to cirrhosis, the cut-off LSM value for significant fibrosis varies depending on the aetiology of the underlying liver disease. ${ }^{(5)}$ In the Singapore population, where CHB is the predominant aetiology, a cut-off LSM value of $9 \mathrm{kPa}$ provides the highest accuracy for the diagnosis of significant fibrosis, allowing correct classification in $73 \%$ of the study cohort. Disease-specific cut-off LSM values can thus be applied in clinical practice to help physicians make management decisions for patients with $\mathrm{CHB}$. In $\mathrm{CHB}$, detection of significant fibrosis guides the decision to commence antiviral treatment to prevent progression to cirrhosis. ${ }^{(15,26)}$ This decision has important economic implications since many patients, especially those with hepatitis B ' $\mathrm{e}$ ' antigen (HBeAg)-negative disease, will require long-term continuous therapy with nucleoside analogues. Conversely, reliable exclusion of significant fibrosis provides the physician with the confidence to delay the initiation of unnecessary and expensive treatment for selected patients. For example, if a 40-year-old Chinese man with HBeAg-negative CHB, ALT 1-2 × ULN and a low-to-moderate hepatitis B virus DNA level (2,000-20,000 IU/mL) has an LSM value $<6 \mathrm{kPa}$, he is unlikely to have significant fibrosis, and can thus be monitored. However, if he has an LSM value $>9 \mathrm{kPa}$, he is likely to have significant fibrosis, which requires treatment to be commenced. Liver biopsy can be avoided in such patients, unless there is a discrepancy between the LSM value and clinical impression. Based on the data from our study, liver biopsy can be avoided in more than $70 \%$ of $\mathrm{CHB}$ patients.
In $\mathrm{CHB}$, fluctuating ALT levels must be taken into account when interpreting LSM values. ${ }^{(29)}$ In our study, we specifically excluded patients with ALT $>5 \times U L N$. Hence, the recommended cut-off LSM value of $9 \mathrm{kPa}$ should only be applied to $\mathrm{CHB}$ patients with ALT within this range. $\mathrm{CHB}$ patients with ALT $>5 \times U L N$ should undergo either repeat TE when the ALT falls below this threshold or liver biopsy for accurate staging of fibrosis. TE algorithms incorporating elevated ALT have been developed, but they have not been widely validated clinically. ${ }^{(30,31)}$

The relatively small number of patients with $\mathrm{CHC}$ and $\mathrm{NASH}$ in our study limits the ability to make definitive conclusions regarding the optimal cut-off LSM values for diagnosis of significant fibrosis in these conditions. Nonetheless, the results from our study are consistent with those of the published literature, demonstrating that the optimal cut-off LSM values for $\mathrm{CHC}$ are similar to those for $\mathrm{CHB}$ (i.e. $9 \mathrm{kPa}$ for significant fibrosis and $12 \mathrm{kPa}$ for cirrhosis). ${ }^{(17)}$ Although the current availability of highly effective, direct-acting antiviral agents has made the diagnosis of significant fibrosis less essential in the management of $\mathrm{CHC}$, TE still has a relevant role to play in patient selection for prioritisation of treatment. $\mathrm{CHC}$ treatment should be prioritised for patients with advanced fibrosis or cirrhosis, justified for those with moderate fibrosis, and individualised for those with no or mild fibrosis. ${ }^{(32)}$ In addition, TE has a role in the non-invasive diagnosis of liver cirrhosis in $\mathrm{CHC}$ patients, and this will have an impact on the choice and duration of the antiviral treatment.

There is currently no pre-existing local data on the reliability of TE in NASH. From our cohort of 51 biopsy-proven NASH patients, we observed that TE was highly reliable for the prediction of significant fibrosis and cirrhosis in NASH patients with AUROCs of 0.907 and 0.950 , respectively. Interestingly, the optimal cutoff LSM values of $11 \mathrm{kPa}$ for significant fibrosis and $15 \mathrm{kPa}$ for cirrhosis are higher in $\mathrm{NASH}$ as compared to $\mathrm{CHB}$ and $\mathrm{CHC}$, and they are also higher compared to other studies conducted on TE in NASH. ${ }^{(14,33)}$ Our results may, however, be confounded by the small sample size, lower prevalence of significant fibrosis in the NASH cohort, and the use of the METAVIR scoring system for grading fibrosis. The METAVIR scoring system was developed and validated for portal-based fibrosis such as viral and alcoholic liver disease, whereas NASH fibrosis should be graded using the $\mathrm{NASH}$-Clinical Research Network grading system. Nonetheless, these interesting results raise the need for larger studies that focus on validating the optimal cut-off LSM values for NASH in Singaporean patients. This is clinically important because the local incidence of $\mathrm{NASH}$ is on the rise, which underscores the necessity of a reliable non-invasive method of assessing fibrosis in NASH.

The strength of the present study lies in the selection of a cohort that meets the recommended quality criteria for TE assessment. ${ }^{(17)}$ Firstly, we selected patients with high-quality TE measurements, with TE performed by experienced operators using standardised methods that strictly adhered to the manufacturer's recommendations. Secondly, we controlled for potential confounders, including elevated BMI, ALT > $5 \times$ ULN and inadequate biopsy samples, which are all known to reduce the reliability of LSM. Thirdly, the inclusion of patients from more 
than one institution reduces the limitations of a single-site study and improves the reliability of our findings. In a sub-analysis comparing the results of the two separate sites, the AUROCs and optimal LSM values for significant fibrosis and cirrhosis were found to be similar.

The main limitation of our study is that it was performed in tertiary centres, which introduces a spectrum bias where study subjects are more likely to have more severe fibrosis. This raises the question of whether the cut-off LSM values in our study can be applied to the use of TE in primary care settings. To answer this question, we have presented a range of cut-off LSM values based on maximal sensitivity, maximal diagnostic accuracy and maximal specificity to aid the clinician in selecting the appropriate cut-off based on the clinical indication for TE (Table III). In the setting of broad-based population screening for liver fibrosis, clinicians may select a cut-off with maximal sensitivity (e.g. LSM > $6 \mathrm{kPa}$ ) to reduce the likelihood of false negatives.

In conclusion, the overall cut-off LSM values of $9 \mathrm{kPa}$ and 13 $\mathrm{kPa}$ provide reasonable accuracy for the prediction of significant fibrosis and cirrhosis, respectively, in the Singapore population. However, the selection of an optimal cut-off LSM value for clinical use should be based on disease-specific cut-off values that vary among different aetiologies of CLD. In chronic viral hepatitis, the optimal cut-off LSM values of $9 \mathrm{kPa}$ (range 6-12 kPa) and $12 \mathrm{kPa}$ (range $6.5-16 \mathrm{kPa}$ ) are identified for the prediction of significant fibrosis and cirrhosis, respectively. The optimal cut-off LSM values for NASH still require further validation. We hope that the results of this prospective, multicentre study in a high-quality cohort will help to standardise the interpretation of TE in Singapore.

\section{ACKNOWLEDGEMENTS}

The authors would like to acknowledge the contributions of all the physicians in the Department of Gastroenterology and Hepatology, Singapore General Hospital and the Division of Gastroenterology and Hepatology, National University Hospital. We also acknowledge the contributions of the technicians who performed the LSM. We thank Dr Jeffrey Ngu Jing Hieng and Dr Roy Soetikno from the Department of Gastroenterology and Hepatology, Singapore General Hospital, for reviewing the manuscript prior to submission. This study was funded by a grant from the Ministry of Health, Singapore (MOH RF Grant RF/HSDP10CS01S).

\section{REFERENCES}

1. D'Amico G, Garcia-Tsao G, Pagliaro L. Natural history and prognostic indicators of survival in cirrhosis: a systematic review of 118 studies. J Hepatol 2006; 44:217-31.

2. Bedossa $P$, Dargère $D$, Paradis $V$. Sampling variability of liver fibrosis in chronic hepatitis C. Hepatology 2003; 38:1449-57.

3. Rousselet MC, Michalak S, Dupré F, et al; Hepatitis Network 49. Sources of variability in histological scoring of chronic viral hepatitis. Hepatology 2005; 41:257-64.

4. Tsochatzis EA, Gurusamy KS, Ntaoula S, et al. Elastography for the diagnosis of severity of fibrosis in chronic liver disease: a meta-analysis of diagnostic accuracy. J Hepatol 2011; 54:650-9.

5. Friedrich-Rust M, Ong MF, Martens S, et al. Performance of transient elastography for the staging of liver fibrosis: a meta-analysis. Gastroenterology 2008; 134:960-74.
6. Talwalkar JA, Kurtz DM, Schoenleber SJ, West CP, Montori VM. Ultrasoundbased transient elastography for the detection of hepatic fibrosis: systematic review and meta-analysis. Clin Gastroenterol Hepatol 2007; 5:1214-20.

7. Degos F, Perez P, Roche B, et al; FIBROSTIC study group. Diagnostic accuracy of FibroScan and comparison to liver fibrosis biomarkers in chronic viral hepatitis: a multicenter prospective study (the FIBROSTIC study). J Hepatol 2010; 53:1013-21.

8. Tapper EB, Castera L, Afdhal NH. FibroScan (vibration-controlled transient elastography): where does it stand in the United States practice. Clin Gastroenterol Hepatol 2015; 13:27-36.

9. Singh S, Fujii LL, Murad MH, et al. Liver stiffness is associated with risk of decompensation, liver cancer, and death in patients with chronic liver diseases: a systematic review and meta-analysis. Clin Gastroenterol Hepatol 2013; 11:1573-84.

10. Castéra L, Vergniol J, Foucher J, et al. Prospective comparison of transient elastography, Fibrotest, APRI, and liver biopsy for the assessment of fibrosis in chronic hepatitis C. Gastroenterology 2005; 128:343-50.

11. Ziol M, Handra-Luca A, Kettaneh A, et al. Noninvasive assessment of liver fibrosis by measurement of stiffness in patients with chronic hepatitis C. Hepatology 2005; 41:48-54.

12. Chang PE, Lui HF, Chau YP, et al. Prospective evaluation of transient elastography for the diagnosis of hepatic fibrosis in Asians: comparison with liver biopsy and aspartate transaminase platelet ratio index. Aliment Pharmacol Ther 2008; 28:51-61.

13. Marcellin $P$, Ziol M, Bedossa $P$, et al. Non-invasive assessment of liver fibrosis by stiffness measurement in patients with chronic hepatitis B. Liver Int 2009; 29:242-7.

14. Wong VW, Vergniol J, Wong GL, et al. Diagnosis of fibrosis and cirrhosis using liver stiffness measurement in nonalcoholic fatty liver disease. Hepatology 2010; 51:454-62.

15. European Association for the Study of the Liver. EASL clinical practice guidelines: management of chronic hepatitis B virus infection. J Hepatol 2012; 57:167-85.

16. Liaw YF, Kao JH, Piratvisuth T, et al. Asian-Pacific consensus statement on the management of chronic hepatitis B: a 2012 update. Hepatol Int 2012; 6:531-61.

17. European Association for Study of Liver; Asociacion Latinoamericana para el Estudio del Higado. EASL-ALEH Clinical Practice Guidelines: non-invasive tests for evaluation of liver disease severity and prognosis. J Hepatol 2015; 63:237-64.

18. Fraquelli M, Rigamonti C, Casazza G, et al. Reproducibility of transient elastography in the evaluation of liver fibrosis in patients with chronic liver disease. Gut 2007; 56:968-73.

19. Castéra L, Foucher J, Bernard PH, et al. Pitfalls of liver stiffness measurement: a 5-year prospective study of 13,369 examinations. Hepatology 2010; 51:828-35.

20. Lucidarme D, Foucher J, Le Bail B, et al. Factors of accuracy of transient elastography (fibroscan) for the diagnosis of liver fibrosis in chronic hepatitis C. Hepatology 2009; 49:1083-9.

21. Arena $U$, Vizzutti $F$, Corti $G$, et al. Acute viral hepatitis increases liver stiffness values measured by transient elastography. Hepatology 2008; 47:380-4.

22. Petta S, Di Marco V, Cammà C, et al. Reliability of liver stiffness measurement in non-alcoholic fatty liver disease: the effects of body mass index. Aliment Pharmacol Ther 2011; 33:1350-60

23. Pang JX, Pradhan F, Zimmer S, et al. The feasibility and reliability of transient elastography using Fibroscan®: a practice audit of 2335 examinations. Can J Gastroenterol Hepatol 2014; 28:143-9.

24. Bedossa P, Poynard T. An algorithm for the grading of activity in chronic hepatitis $C$. The METAVIR Cooperative Study Group. Hepatology 1996; 24:289-93.

25. Wai CT, Greenson JK, Fontana RJ, et al. A simple noninvasive index can predict both significant fibrosis and cirrhosis in patients with chronic hepatitis C. Hepatology 2003; 38:518-26.

26. Lok AS, McMahon BJ. Chronic hepatitis B: update 2009. Hepatology 2009; 50:661-2.

27. Göbel T, Schadewaldt-Tümmers J, Greiner L, et al. Transient elastography improves detection of liver cirrhosis compared to routine screening tests. World J Gastroenterol 2015; 21:953-60.

28. Choong CC, Venkatesh SK, Siew EP. Accuracy of routine clinical ultrasound for staging of liver fibrosis. J Clin Imaging Sci 2012; 2:58

29. Coco B, Oliveri F, Maina AM, et al. Transient elastography: a new surrogate marker of liver fibrosis influenced by major changes of transaminases. J Viral Hepat 2007; 14:360-9.

30. Chan HL, Wong GL, Choi PC, et al. Alanine aminotransferase-based algorithms of liver stiffness measurement by transient elastography (Fibroscan) for liver fibrosis in chronic hepatitis B. J Viral Hepat 2009; 16:36-44.

31. Wong VW, Lampertico P, de Lédinghen V, et al. Probability-based interpretation of liver stiffness measurement in untreated chronic hepatitis B patients. Dig Dis Sci 2015; 60:1448-56.

32. European Association for the Study of the Liver. EASL recommendations on treatment of hepatitis C 2014. J Hepatol 2014; 61:373-95.

33. Yoneda M, Yoneda $M$, Mawatari $H$, et al. Noninvasive assessment of liver fibrosis by measurement of stiffness in patients with nonalcoholic fatty liver disease (NAFLD). Dig Liver Dis 2008; 40:371-8. 